\title{
Aerobic Oxidation of Alcohols to Carbonyl Compounds Catalyzed by N-Hydroxyphthalimide (NHPI) Combined with CoTPP-Zn 2 Al-LDH
}

\author{
WEIYOU ZHOU, DONGWEI CHEN, AIJUN CUI, JUNFENG QIAN, MINGYANG HE* and \\ QUN CHEN \\ Jiangsu Key Laboratory of Advanced Catalytic Materials and Technology, Changzhou University, \\ Changzhou 213164, China \\ Email: hemy_cczu@126.com
}

MS received 18 October 2016; revised 19 January 2017; accepted 19 January 2017

\begin{abstract}
A catalytic system for the aerobic oxidation of alcohols by N-hydroxyphthalimide (NHPI) combined with cobalt porphyrin intercalated heterogeneous hybrid catalyst (CoTPP- $\mathrm{Zn}_{2} \mathrm{Al}-\mathrm{LDH}$ ) has been developed. The results showed that this catalytic system can effectively catalyze the oxidation of alcohols to the corresponding carbonyl compounds. And the hybrid catalyst can be reused for five times with no appreciable reduction of activity.
\end{abstract}

Keywords. Hydrotalcite-like compounds; cobalt porphyrin; alcohol oxidation; N-hydroxyphthalimide.

\section{Introduction}

The selective oxidation of alcohols to the corresponding carbonyl compounds is one of the most important reactions in organic synthesis and fine chemical industries. ${ }^{1}$ Numerous methods have been developed for the transformation using varied oxidants, such as $\mathrm{KMnO}_{4}, \mathrm{H}_{2} \mathrm{O}_{2}$, tbutylhydroperoxide, $m$-chloroperbenzoic acid, etc. From the sustainable and environmental viewpoints, development of efficient methodology for the oxidation using molecular oxygen as the terminal oxidant without a stoichiometric amount of additives is very attractive.

Since Ishii et al., first found that NHPI could efficiently catalyze the aerobic oxidation of various organic substrates to oxygen containing compounds, ${ }^{2}$ an efficient catalytic method for the low temperature oxygenation of alcohol with dioxygen was developed by using NHPI as a catalyst and a metal salt as co-catalyst. Actually, the co-catalyst plays an important role in accelerating the reaction and affecting the selectivity. Some catalysts, including cobalt salts, ${ }^{3}\left[\mathrm{Bu}_{4} \mathrm{~N} \mathrm{VO}_{3},{ }^{4}\right.$ $\mathrm{VO}(\mathrm{acac})_{2},{ }^{5}$ copper salts, ${ }^{6}$ iron salt, ${ }^{7}$ ceric ammonium nitrate, ${ }^{8}$ acetaldehyde, ${ }^{9}$ ammonium salts ${ }^{10}$ and $\mathrm{NO}_{2},{ }^{11}$ have been investigated in the reaction, but the homogeneous nature of these catalytic systems leads to the difficulty of recovery of the catalyst and complexity in the process.

In the practical point of view, development of heterogeneous co-catalyst for the NHPI/O $\mathrm{O}_{2}$ system for the

\footnotetext{
*For correspondence
}

selective oxidation of alcohol to carbonyl compound is still keenly desirable. To the best of our knowledge, only a few heterogeneous catalytic systems have been reported cobalt porphyrin (CoTPP) intercalated $\mathrm{ZnAl}$ hydrotalcite (CoTPP- $\mathrm{Zn}_{2} \mathrm{Al}-\mathrm{LDH}$ ) is an efficient heterogeneous catalyst for the oxidation of ketone and alcohol, ${ }^{13}$ where excess additives were needed. In this paper, we are reporting the aerobic oxidation of alcohols by a catalytic system, consisting of NHPI and CoTPP-Zn ${ }_{2}$ Al-LDH hybrid.

\section{Experimental}

\subsection{Materials}

All reagents and solvents were of analytical grade and were obtained commercially. CoTPP- $\mathrm{Zn}_{2} \mathrm{Al}-\mathrm{LDH}$ was prepared according to our previous reported procedure. ${ }^{13 \mathrm{~b}}$ Quantitative determination of the CoTPP content in the hybrid was performed byinductively coupled plasma analysis (ICP, Varian Vista-AX device). Typically, a CoTPP loading at $c a .0 .38 \mathrm{mmol} \mathrm{g}^{-1}$ was obtained. No impurities were found in the cyclohexanol by GC-MS (Shimadzu GCMS-2010) analysis before use.

\subsection{General procedure for the aerobic oxidation of alcohols using $\mathrm{NHPI} / \mathrm{O}_{2} / \mathrm{CoTPP}-\mathrm{Zn}_{2} \mathrm{Al}-\mathrm{LDH}$ system}

In a typical experiment, a solution of NHPI $(0.6 \mathrm{mmol}$, $20 \mathrm{~mol} \%)$ and CoTPP- $\mathrm{Zn}_{2} \mathrm{Al}-\mathrm{LDH} \quad(0.01 \mathrm{mmol}$ of CoTPP content, $30 \mathrm{mg})$ in $\mathrm{PhCN}$ or $\mathrm{CH}_{3} \mathrm{CN}(10 \mathrm{~mL})$ 
was prepared in a $25 \mathrm{~mL}$ round-bottom flask equipped with magnetic stirrer. To this solution alcohol ( $3 \mathrm{mmol})$ was added and the resulting mixture was stirred at 100 or $80^{\circ} \mathrm{C}$. Then dioxygen was bubbled through the solution at a rate of $20 \mathrm{~mL} \mathrm{~min}^{-1}$. All the products were identified by GC-MS and quantified at different intervals during the reaction by GC-FID (Shimadzu GC-2010AF).

\section{Results and Discussion}

In this study, cyclohexanol was selected as the model substrate to optimize the reaction conditions. Firstly, various solvents including dichloroethane (DCE), dioxane (Diox), dimethyl formamide (DMF), acetonitrile $(\mathrm{ACN})$, and cyanobenzene $(\mathrm{PhCN})$, were optimized. With DMF as the solvent, much lower conversion of cyclohexanol was obtained. When dioxane was used as the solvent, the oxidation product from dioxane was observed. Other three solvents exhibited similar activities at $80^{\circ} \mathrm{C}$, including low boiling solvents, ACN and DCE. We then investigated the effect of reaction temperature using $\mathrm{PhCN}$ as the solvent. Decreasing the temperature significantly reduced the conversion. $>90 \%$ of conversion could be obtained when the temperature reached $100^{\circ} \mathrm{C}$. After optimization of the amounts of NHPI and the catalyst, a high yield of $91 \%$ cyclohexanone was obtained without any other byproduct. The blank reaction without catalyst showed low conversion (Table 1, entry 12), indicating that the prepared hybrid material performed as catalyst in the reaction.

It has been reported that the phthalimide $\mathrm{N}$-oxyl (PINO) radical is produced from the reaction of $\mathrm{O}_{2}$ and
NHPI in the presence of metal co-catalyst. ${ }^{12}$ In the alcohol oxidation catalyzed by NHPI, the radical is able to abstract a hydrogen atom from the alcohol and accelerate the transformation. We suppose that in the alcohol oxidation catalyzed by NHPI and CoTPP-Zn ${ }_{2} \mathrm{Al}-\mathrm{LDH}$ as a co-catalyst, PINO radical would be produced in the oxidation of NHPI by $\mathrm{O}_{2}$ and the hybrid.

Under the optimized reaction conditions, the scope of the substrates was then examined. Secondary alcohols were firstly investigated in the study. The results (Table 2, entries 2-5) clearly show that both benzylic and aliphatic alcohols can be efficiently oxidized to their corresponding ketones with excellent conversions and yields, markedly higher than the previous reported results. ${ }^{12}$ In addition, no other by-products were observed in the oxidation of secondary alcohols, indicating the present system is an efficient method for the selective oxidation secondary alcohols. For the primary alcohols, both aldehyde and acid were observed as the products, because aldehyde was easily oxidized into the corresponding carboxylic acid with high conversion and yield. ${ }^{12 \mathrm{c}}$ To obtain the higher yield of the corresponding aldehyde, ACN was employed as the solvent and the reaction temperature was decreased to $80^{\circ} \mathrm{C}$. In the oxidation of benzyl alcohol, moderate conversion and selectivity were obtained under the preliminary optimized conditions (Table 2, entry 6). Among the aliphatic primary alcohols and the aromatic alcohols with hetero atoms, such as 3-furanmethanol, pyridine-2-methanol and 3-thiophenemethanol, moderate yields of the corresponding aldehydes could also been obtained. It should be noted that, compared with the results without CoTPP- $\mathrm{Zn}_{2} \mathrm{Al}-\mathrm{LDH}$, the conversion of the substrates increased under the catalysis with the

Table 1. Oxidation of cyclohexanol catalyzed by NHPI/O ${ }_{2} / \mathrm{CoTPP}-\mathrm{Zn}_{2} \mathrm{Al}-\mathrm{LDH}$ system.

\begin{tabular}{lccccc}
\hline Entry & Solvent & $\mathrm{T}\left({ }^{\circ} \mathrm{C}\right)^{\mathrm{a}}$ & NHPI $(\mathrm{mol} \%)$ & $\mathrm{m}(\mathrm{mg})^{\mathrm{b}}$ & $\mathrm{C}(\%)^{\mathrm{c}}$ \\
\hline 1 & ACN & 80 & 20 & 30 & 41 \\
2 & Diox & 80 & 20 & 30 & 81 \\
3 & DMF & 80 & 20 & 30 & $<1$ \\
4 & DCE & 80 & 20 & 30 & 66 \\
5 & PhCN & 80 & 20 & 30 & 45 \\
6 & PhCN & 100 & 20 & 30 & 91 \\
7 & PhCN & 60 & 20 & 30 & 33 \\
8 & PhCN & 100 & 20 & 15 & 78 \\
9 & PhCN & 100 & 20 & 40 & 93 \\
10 & PhCN & 100 & 15 & 30 & 87 \\
11 & PhCN & 100 & 10 & 30 & 83 \\
12 & PhCN & 100 & 20 & 0 & 68 \\
\hline
\end{tabular}

Reaction conditions: cyclohexanol $3 \mathrm{mmol}$, solvent $10 \mathrm{~mL}, \mathrm{O}_{2} 20 \mathrm{~mL} / \mathrm{min}, 24 \mathrm{~h}$.

${ }^{\mathrm{a}}$ Reaction temperature; ${ }^{\mathrm{b}}$ Amount of catalyst; ${ }^{\mathrm{c}}$ Conversion of cyclohexanol; no other by-product was observed. 
Table 2. Catalytic oxidation of alcohol by NHPI/O ${ }_{2} / \mathrm{CoTPP}-\mathrm{Zn}_{2} \mathrm{Al}-\mathrm{LDH}$ system.

\begin{tabular}{|c|c|c|c|c|c|c|c|}
\hline Entry & Substrate & Product & Solvent & $\mathrm{T}\left({ }^{\circ} \mathrm{C}\right)^{\mathrm{a}}$ & $\mathrm{t}(\mathrm{h})^{\mathrm{b}}$ & $\mathrm{C}(\%)^{\mathrm{c}}$ & $\mathrm{S}(\%)^{\mathrm{d}}$ \\
\hline 1 & & & $\mathrm{PhCN}$ & 100 & 24 & $91(68)$ & $>99$ \\
\hline 2 & & & $\mathrm{PhCN}$ & 100 & 28 & $93(77)$ & $>99$ \\
\hline 3 & & & $\mathrm{PhCN}$ & 100 & 30 & $92(80)$ & $>99$ \\
\hline 4 & & & $\mathrm{PhCN}$ & 100 & 28 & $91(78)$ & $>99$ \\
\hline 5 & & & $\mathrm{ACN}$ & 80 & 30 & $88(75)$ & $>99$ \\
\hline 6 & & & $\mathrm{ACN}$ & 80 & 7 & $62(40)$ & $77(56)$ \\
\hline 7 & & & $\mathrm{ACN}$ & 80 & 16 & $82(51)$ & $74(46)$ \\
\hline 8 & & & $\mathrm{ACN}$ & 80 & 20 & $96(78)$ & $92(78)$ \\
\hline 9 & & & $\mathrm{ACN}$ & 80 & 10 & $75(56)$ & $56(44)$ \\
\hline 10 & & & $\mathrm{ACN}$ & 80 & 12 & $82(69)$ & $36(13)$ \\
\hline 11 & & & $\mathrm{ACN}$ & 80 & 44 & $72(10)$ & $91(81)$ \\
\hline 12 & & & $\mathrm{ACN}$ & 80 & 24 & $45(10)$ & $95(80)$ \\
\hline 13 & & & $\mathrm{ACN}$ & 80 & 44 & $79(12)$ & $90(82)$ \\
\hline
\end{tabular}

Reaction conditions: alcohol $3 \mathrm{mmol}$, solvent $10 \mathrm{~mL}, \mathrm{O}_{2} 20 \mathrm{~mL} / \mathrm{min}$, NHPI $0.6 \mathrm{mmol}$, CoTPP- $\mathrm{Zn}_{2} \mathrm{Al}-\mathrm{LDH} 30 \mathrm{mg}$. All the products were identified by GC-MS. ${ }^{\mathrm{a}}$ Reaction temperature; ${ }^{\mathrm{b}}$ Reaction time; ${ }^{\mathrm{c}}$ Conversion, value in parenthesis shows the result without CoTPP- $\mathrm{Zn}_{2} \mathrm{Al}-\mathrm{LDH} ;{ }^{\mathrm{d}}$ Selectivity, value in parenthesis shows the result without CoTPP- $\mathrm{Zn}_{2} \mathrm{Al}-\mathrm{LDH}$.

hybrid, indicating the catalytic function of the material. On the other hand, the selectivity of the aldehyde increased to some extent when the primary alcohols were used as the substrates, which were obviously higher than the reported results over NHPI/O ${ }_{2}$ systems. ${ }^{12}$ The increase in the selectivity may be due to the basicity of the hydrotalcites catalyst, ${ }^{13 b, 14}$ which is a representative basic material. ${ }^{15}$

Subsequently, the recyclability of the CoTPP- $\mathrm{Zn}_{2} \mathrm{Al}-$ LDH was examined in the aerobic oxidation of cyclohexanol by NHPI. The catalyst showed no appreciable reduction of activity even after five runs 


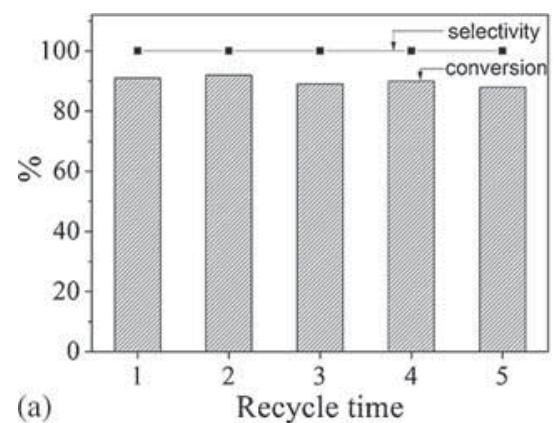

(a)

Figure 1. (A) The recyclability of the catalyst CoTPP- $\mathrm{Zn}_{2} \mathrm{Al}-\mathrm{LDH}$ catalyst; (B) XRD pattern of recycled and fresh catalyst. Reaction conditions: alcohol $3 \mathrm{mmol}$, solvent $10 \mathrm{~mL}, \mathrm{O}_{2} 20 \mathrm{~mL} / \mathrm{min}$, NHPI $0.6 \mathrm{mmol}$, CoTPP-Zn ${ }_{2} \mathrm{Al}-\mathrm{LDH}$ $30 \mathrm{mg}$.
(Figure 1A), and the selectivities were above $99 \%$. XRD pattern for the reused catalyst suggested that the layered structure was completely preserved after several reuses (Figure 1B).

\section{Conclusions}

In this work, aerobic oxidation of alcohols has been investigated by NHPI combined with CoTPPintercalated hydrotalcites. The results indicated that CoTPP- $\mathrm{Zn}_{2} \mathrm{Al}-\mathrm{LDH}$ is an efficient catalyst for the oxidation of alcohols to corresponding carbonyl compounds through $\mathrm{O}_{2} / \mathrm{NHPI}$ system and tolerates a wide range of substrates. The catalyst recycling test suggested that the CoPcTs- $\mathrm{Zn}_{2} \mathrm{Al}-\mathrm{LDH}$ is durable and quite good under the tested reaction conditions. The work on the development of more efficient catalysts based on the hybrid and structure-activity relationship is in progress.

\section{Acknowledgements}

This work was supported by the National Natural Science Foundation of China (Grant No. 21403018) and Prospective Joint Research Project on the Industry, Education and Research of Jiangsu Province (BY2014037-17).

\section{References}

1. (a) Caron S, Dugger R W, Ruggeri S G, Ragan J A and Ripin D H B 2006 Large-scale oxidations in the pharmaceutical industry Chem. Rev. 106 2943; (b) Delaude $\mathrm{L}$ and Laszlo P 1996 A novel oxidizing reagent based on potassium ferrate (VI) 1 J. Org. Chem. 61 6360; (c) Mahdavi V and Mardani M 2012 Selective oxidation of benzyl alcohol with tert-butylhydroperoxide catalysed via Mn (II) 2, 2-bipyridine complexes immobilized over the mesoporous hexagonal molecular sieves (HMS) J. Chem. Sci. 124 1107; (d) Wang Z G, Jin Y, Cao X H and Lu M 2014 Bi-functionalized PEG $_{1000}$

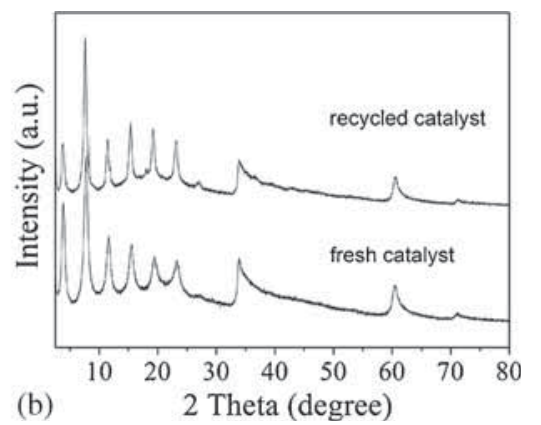

ionic liquid [Imim- $\left.\mathrm{PEG}_{1000}-\mathrm{TEMPO}\right]\left[\mathrm{CuCl}_{2}^{-}\right]$: An efficient and reusable catalytic system for solvent-free aerobic oxidation of alcohols New J. Chem. 38 4149; (e) Shojaei A F, Tabatabaeian K, Zanjanchi M A, Moafi H F and Modirpanah N 2015 Synthesis, characterization and study of catalytic activity of Silver doped $\mathrm{ZnO}$ nanocomposite as an efficient catalyst for selective oxidation of benzyl alcohol J. Chem. Sci. 127481

2. Ishii $Y$, Nakayama $K$, Takeno $M$, Sakaguchi S, Iwahama T and Nishiyama Y 1995 Novel catalysis by $\mathrm{N}$-hydroxyphthalimide in the oxidation of organic substrates by molecular oxygen $\mathrm{J}$. Org. Chem. 603934

3. (a) Iwahama $\mathrm{T}$, Yoshino Y, Keitoku T, Sakaguchi S and Ishii Y 2000 Efficient oxidation of alcohols to carbonyl compounds with molecular oxygen catalyzed by N-hydroxyphthalimide combined with a Co species J. Org. Chem. 65 6502; (b) Iwahama T, Sakaguchi S, Nishiyama Y and Ishii Y 1995 Aerobic oxidation of alcohols to carbonyl compounds catalyzed by N-hydroxyphthalimide (NHPI) combined with $\mathrm{Co}(\mathrm{acac})_{3}$ Tetrahedron Lett. 36 6923

4. (a) Figiel P J and Sobcz ak J M 2007 Aerobic oxidation of alcohols and alkylaromatics with dioxygen catalysed by $\mathrm{N}$-hydroxyphthalimide with vanadium co-catalysts New J. Chem. 31 1668; (b) Figiel P J and Sobczak J M 2009 Mechanistic investigation of the catalytic system based on N-hydroxy-phthalimide with vanadium cocatalysts for aerobic oxidation of alcohols with dioxygen J. Catal. 263167

5. Figiel P J, Sobczak J M and Ziółkowski J J 2004 New efficient aerobic oxidation of some alcohols with dioxygen catalyzed by $\mathrm{N}$-hydroxyphtalimide with vanadium co-catalysts Chem. Commun. 244

6. (a) Chen B, Li J, Yang G and Gao S 2015 Aerobic oxidation of benzyl alcohols using a novel combination of $\mathrm{N}$-hydroxyphthalimide (NHPI) with $\mathrm{HNO}_{3}$ and $\mathrm{CuBr}_{2}$ Res. Chem. Intermed. 41 3929; (b) Yang G, Wang L, Li J, Zhang Y, Dong X L, Lv Y and Gao S 2012 Aerobic oxidation of alcohols using a novel combination of N-hydroxyphthalimide (NHPI) and $\mathrm{CuBr}$ Res. Chem. Intermed. 38 775; (c) Kompanets $\mathrm{M} \mathrm{O}$, Kushch O V, Litvinov Y E, Pliekhov O L, Novikova K V, Novokhatko A O, Shendrik A N, Vasilyev A V and Opeida I O 
2014 Oxidation of 5-hydroxymethylfurfural to 2,5diformylfuran with molecular oxygen in the presence of N-hydroxyphthalimide Catal. Commun. 5760

7. Zhao H Q, Sun W, Miao C X and Zhao Q Y 2014 Aerobic oxidation of secondary alcohols using NHPI and iron salt as catalysts at room temperature J. Mol. Catal. A: Chem. 39362

8. Kim S S and Rajagopal G 2004 Efficient aerobic oxidation of alcohols to carbonyl compounds with NHPI/CAN catalytic system Synth. Commun. 342237

9. Einhorn C, Einhorn J, Marcadal C and Pierre J L 1997 Oxidation of organic substrates by molecular oxygen mediated by N-hydroxyphthalimide (NHPI) and acetaldehyde Chem. Commun. 447

10. Figiel P J and Sobczak J M 2001 Unexpected catalytic activity of $\mathrm{N}$-hydroxyphthalimide combined with some co-catalyst in oxidation of organic substrates by dioxygen Pol. J. Chem. 75869

11. Sakaguchi S, Shibamoto A and Ishii Y 2002 Remarkable effect of nitrogen dioxide for $\mathrm{N}$-hydroxyphthalimidecatalyzed aerobic oxidation of methylquinolines Chem. Commun. 180

12. (a) Monfared H H, Meyer H and Janiak C 2013 Dioxygen oxidation of 1-phenylethanol with gold nanoparticles and N-hydroxyphthalimide in ionic liquid $\mathrm{J}$. Mol. Catal. A: Chem. 372 72; (b) Rajabi F and Karimi B 2005 Efficient aerobic oxidation of alcohols using a novel combination N-hydroxy phthalimide (NHPI) and a recyclable heterogeneous cobalt complex J. Mol. Catal. A: Chem. 232 95; (c) Zahed B and Monfared H H 2015 A comparative study of silver-graphene oxide nanocomposites as arecyclable catalyst for the aerobic oxidation of benzyl alcohol: support effect Appl. Surf. Sci. 328536

13. (a) Zhou W Y, Tian P, Sun F A, He MY and Chen Z X 2015 Efficient catalysis of the aerobic Baeyer-Villiger oxidation over a bifunctional catalyst based on cobalt tetraphenylporphyrin intercalated into $\mathrm{Zn}_{2} \mathrm{Al}$ hydrotalcite Asian J. Org. Chem. 433; (b) Zhou W Y, Tian P, Sun F A, He M Y and Chen Q 2016 Highly efficient transformation of alcohol to carbonyl compounds under a hybrid bifunctional catalyst originated from metalloporphyrins and hydrotalcite J. Catal. 335105

14. (a) Song H, Kang B and Hong S H 2014 Fe-catalyzed acceptorless dehydrogenation of secondary benzylic alcohols ACS Catal. 4 2889; (b) Tsunoyama H, Ichikuni N, Sakurai H and Tsukuda T 2009 Effect of electronic structures of $\mathrm{Au}$ clusters stabilized by poly(N-vinyl-2pyrrolidone) on aerobic oxidation catalysis J. Am. Chem. Soc. 131 7086; (c) Amini M, Najafpour M M, Zare $\mathrm{M}$ and Amini E 2014 Nanolayered manganese-calcium oxide as an efficient and environmentally friendly catalyst for alcohol oxidation J. Mol. Catal. A: Chem. 394 303

15. (a) Wang Z, Fongarland P, Lu G Z and Essayem N 2014 Reconstructed La-, Y-, Ce-modified MgAl-hydrotalcite as a solid base catalyst for aldol condensation: Investigation of water tolerance J. Catal. 318 108; (b) Debecker D P, Gaigneaux E M and Busca G 2009 Exploring, tuning, and exploiting the basicity of hydrotalcites for applications in heterogeneous catalysis Chem. Eur. J. 15 3920 